\title{
Hospital outcomes associated with new-onset atrial fibrillation during ICU admission: A multicentre competing risks analysis
}

Jonathan P Bedford., MSc, MRCP*, Jonathan Bedford@ndcn.ox.ac.uk;

Stephen Gerry., MSc, Stephen.Gerry@CSM.ox.ac.uk²;

Robert A Hatch., BA, FRCA, Robert.Hatch@ndcn.ox.ac.uk';

Ian Rechner., BSc, FRCA, Ian.Rechner@royalberkshire.nhs.uk³;

J Duncan Young., DM, FRCA, Duncan.Young@ndcn.ox.ac.uk;

Peter J Watkinson., MD, Peter.Watkinson@ndcn.ox.ac.uk ${ }^{1}$

${ }^{1}$ Nuffield Department of Clinical Neurosciences, University of Oxford, Oxford, UK

${ }^{2}$ Centre for Statistics in Medicine, Nuffield Department of Orthopaedics, Rheumatology \& Musculoskeletal Sciences,

University of Oxford, Oxford, UK

${ }^{3}$ Department of Intensive Care Medicine, Royal Berkshire Hospital, Reading, UK

*Corresponding author. Kadoorie Centre for Critical Care Research and Education, level 3, John Radcliffe Hospital, Headley Way, Headington, Oxford, UK, OX3 9DU. Tel. +441865231447. Email: Jonathan.Bedford@ndcn.ox.ac.uk ORCID: 0000-0001$9455-022 X$

Word count: 3000 


\section{Abstract}

Purpose

New onset atrial fibrillation (NOAF) in critically ill patients has been associated with increased shortterm mortality. Analyses that do not take into account the time-varying nature of NOAF can underestimate its association with hospital outcomes.

We investigated the prognostic association of NOAF with hospital outcomes using competing risks methods.

\section{Materials and Methods}

We undertook a retrospective cohort study in three general adult intensive care units (ICUs) in the UK from June 2008 to December 2015. We excluded patients with known prior atrial fibrillation or an arrhythmia within four hours of ICU admission.

To account for the effect of NOAF on the rate of death per unit time and the rate of discharge alive per unit time we calculated subdistribution hazard ratios (SDHRs).

Results

Of 7541 patients that fulfilled our inclusion criteria, 831 (11.0\%) developed NOAF during their ICU admission. NOAF was associated with an increased duration of hospital stay (CSHR $0.68(95 \% \mathrm{Cl}$ 0.63-0.73)) and an increased rate of in-hospital death per unit time (CSHR 1.57 (95\% $\mathrm{Cl} 1.37-1.1 .81)$ ). This resulted in a strong prognostic association with dying in hospital (adjusted SDHR 2.04 (1.79 2.32)). NOAF lasting over 30 minutes was associated with increased hospital mortality.

\section{Conclusions}

Using robust methods we demonstrate a stronger prognostic association between NOAF and hospital outcomes than previously reported. 
Keywords

Atrial fibrillation

Arrhythmia

Critical care

Mortality

Outcomes

Competing risks

\section{List of abbreviations}

AF: atrial fibrillation

CSHR: cause-specific hazard ratio

ICU: intensive care unit

LOS: length of stay

NOAF: new-onset atrial fibrillation

SDHR: subdistribution hazard ratio 


\section{Introduction}

New-onset atrial fibrillation (NOAF) is a common arrhythmia in patients treated on an intensive care unit (ICU) [1]. It occurs in $4.5-11 \%$ of all patients [2-6], increasing to $23 \%$ in patients with septic shock [7].

NOAF in patients treated on an ICU is associated with increased mortality $[1,4]$. Patients with a greater severity of illness and a poorer prognosis are more likely to develop NOAF $[2,8]$. NOAF may therefore be a marker of disease severity without an independent influence on mortality [9]. The debate over the attributable mortality of NOAF is ongoing and some studies have not demonstrated an independent association after correcting for disease severity $[10,11]$. There is however a growing body of evidence demonstrating an association with in-ICU $[12,13]$, hospital [14-18] and long-term $[12,14,19]$ mortality after correcting for confounding variables. It is not clear how the duration of NOAF whilst in ICU is associated with hospital outcomes.

The aim of this study was to investigate the association of NOAF during an ICU admission with hospital outcomes. We hypothesised that NOAF during ICU admission is independently associated with worse hospital outcomes when taking into account the time-varying nature of NOAF.

\section{Materials and Methods}

\section{Study design}

We performed a retrospective analysis of patient data collected for the Post Intensive Care Riskadjusted Alerting and Monitoring (PICRAM) database [20]. This study was granted ethical approval by the NRES Committee Oxford (ref: 11/SC/0440) and the National Information Governance Board (ref: ECC 7-05(f)/2011). The PICRAM database was created with retrospectively collected data from the ICU clinical information system relating to patients treated on three general ICUs in the UK from 2008 to 2015 . These included one 8-bed and two 16-bed mixed medical / surgical ICUs. The PICRAM database contains comprehensive data relating to patient demographics, vital signs, interventions 
and investigation results. We adhered to the Strengthening the Reporting of Observational Studies in Epidemiology (STROBE) guidelines [21].

\section{Study population}

We included data relating to all adult ( $\geq 16$ years) patients during their first ICU admission. Sites included three mixed medical-surgical intensive care units in Oxford and Reading. These units did not admit patients after cardiac surgery. We excluded patients with missing hospital outcome data. We also excluded patients with an ICU length of stay (LOS) of less than 24 hours. These patients were excluded in line with similar studies [22] as they were typically admitted after elective surgery and were not representative of a critically ill population.

To exclude patients with pre-existing arrhythmias we identified any entry of arrhythmias in the medical history or any record of medications prescribed for the purpose of heart rhythm management in the medication history. Drug indications were routinely recorded allowing classification in the cases of medications with multiple potential indications. We also excluded patients with any recording of significant arrhythmia in the first four hours of arrival to ICU. This served as another mechanism to exclude patients with pre-admission AF, as the start time of arrhythmias can be unclear in the initial stages of ICU admission [23].

\section{Covariates and outcomes}

Three-lead electrocardiogram was displayed continuously and heart rhythm was recorded by the bedside nurse at regular intervals. There is no consensus definition of NOAF during critical illness [24]. Due to our interest in brief episodes of $A F$, we defined new-onset $A F$ as the documentation of atrial fibrillation or atrial flutter lasting for 30 seconds or more [25]. We included atrial flutter in line with previous studies $[5,13,18]$ as differentiation from atrial fibrillation can be difficult and management strategies are similar. A documented heart rhythm was assumed to persist until the next rhythm was recorded. The primary endpoints of this study were hospital mortality and hospital discharge alive. Missing admission covariates were imputed using multiple imputation [26]. 


\section{Statistical analysis}

We aimed to study whether NOAF during ICU admission is independently associated with worse hospital outcomes and to study the impact of NOAF of different durations.

Continuous variables were summarised as median [interquartile range] and categorical variables summarised as number (percentages). We visually assessed data normalcy using quantile-quantile plots. All statistical analysis was performed using R Core v3.6.1 [27].

\section{Adjustment for confounding}

To analyse the association of NOAF with hospital outcomes, we adjusted for admission covariates.

We adjusted for 15 severe comorbidities (liver cirrhosis, portal hypertension, hepatic

encephalopathy, severe cardiovascular disease (New York Heart Association Class IV), severe respiratory disease (permanent shortness of breath with light activity due to pulmonary disease), home ventilation, chronic renal replacement therapy, HIV/AIDS, steroid therapy, radiotherapy, chemotherapy, metastatic disease, acute or chronic leukaemia and lymphoma). Although relatively rare, the presence of one or more of these has been shown to be independently associated with mortality in ICU patients [28] . Additionally we adjusted for patient sex and the OASIS score [29]. The OASIS score is an admission illness severity score developed to predict ICU and hospital mortality. It consists of age, elective surgery, pre-ICU LOS and seven physiological measurements. Age and physiological measurements were therefore not included as individual covariates in our analysis. Covariates were included when adjusting, regardless of association with outcome to avoid bias introduced by variable selection methods [30].

Sensitivity analysis

Further to our primary sex, comorbidity and OASIS score-adjusted model, we constructed three further models to test the robustness of our results. Model 2 included the OASIS score only. Model 3 included all features of our primary model plus admission laboratory values (potassium, magnesium, urea, creatinine, white cell count and lactate) selected for their association with patient outcomes during critical illness [13, 14, 31-35]. Model 4 included all features of model 3 plus the use of 
vasoactive medications. We also repeated our primary analysis using an alternative NOAF definition potentially relevant to critically ill patients, namely atrial fibrillation or atrial flutter lasting for $\geq 1 \mathrm{~h}$ or requiring pharmacological therapy or requiring cardioversion $[13,36]$.

\section{Hospital outcome}

We used multivariable competing risks analysis to assess the independent association between NOAF and the dichotomous outcomes of hospital mortality and hospital discharge alive.

\section{NOAF AS A TIME-VARYING COVARIATE}

A patient who dies early has less time to develop NOAF, so will be more likely to be classified into the non-AF cohort. Similarly patients who survive longer have more opportunity to develop NOAF $[37,38]$. We therefore analysed NOAF as a time-varying covariate to avoid early deaths inflating the risk of mortality in the non-AF cohort [39].

\section{HOSPITAL DISCHARGE AS A COMPETING RISK TO MORTALITY}

Traditional survival analysis assumes non-informative censoring i.e. the hazard of death remaining unchanged when a censoring event occurs [40]. However, discharged patients have a different risk of death than those that remain on the ICU $[41,42]$. Hospital discharge should therefore not be treated as a censoring event, but rather a competing risk to mortality. Competing risks are events during follow-up that either prevent the primary outcome from occurring, or alter its probability [43]. In the presence of competing risks, appropriate methodology should be used [42, 44]. We therefore firstly generated cause-specific hazard ratios to estimate the aetiological association of NOAF with ICU mortality and on ICU discharge alive. The cause-specific hazard ratio can be considered a measure of aetiological association i.e. the degree with which the occurrence of NOAF is directly associated with hospital mortality rate or rate of hospital discharge [45].

We calculated the subdistribution hazard ratio (SDHR) to estimate the prognostic association between NOAF and hospital outcomes. Cause-specific hazards are appropriate for addressing questions of aetiology. Questions of prognosis and individual risk (e.g. regardless of the direct 
association, whether a patient who experience NOAF during an ICU admission is more likely to die in hospital) are better addressed using subdistribution hazard-based methods [46-48]. The SDHR estimates the effects of a variable on the cumulative incidence of an outcome in the presence of competing risks. It constitutes a composite measure of risk that jointly evaluates the aetiological association of a risk factor with the outcome of interest and of the risk factor with all competing outcomes [49].

\section{NOAF DURATION AND ASSOCIATED MORTALITY}

To analyse the independent impact of NOAF of different durations on hospital outcome, we performed multivariable logistic regression with NOAF duration as a categorical variable. This method was selected to avoid survivorship bias that would be introduced by longer episodes of NOAF. We adjusted for the same variables used in the survival analysis.

\section{Results}

\section{Study population}

The PICRAM dataset includes data relating to 12288 index admissions. Hospital outcome data were not available for 531 (4.3\%) of admissions. Missing hospital outcome data was due to incomplete linkage of two databases and was not related to patient characteristics. A comparison of available data from patients with and without missing outcome data did not reveal any systematic differences (see Supplementary table 1, Additional file 1). Of the remaining 11757 admissions, 2870 had an ICU LOS of less than 24 hours. We identified 1346 of the remaining patients with pre-existing AF or AF occurring in the first 4 hours. Of the 7541 patients that fulfilled our inclusion criteria, $831(11.0 \%)$ developed NOAF during their admission.

No data were missing in our cohort for baseline demographic or comorbidity variables. After exclusion of patients with brief ICU stays, no data were missing for physiological variables or heart rhythm. Laboratory data within the first 24 hours was not complete, with $2.43 \%$ values missing 
overall (range $0.25-3.65 \%$ for individual variables). Details of missing laboratory data and multiple imputation can be found in Supplementary table 2, Additional file 1.

Summary patient characteristics are displayed in table 1. Patients with NOAF appeared older, more unwell and more likely to be non-elective admissions.

Table 1 - Demographic and clinical characteristics of included patients.

$\begin{array}{rrr}\text { Variable } & \text { Never AF } & \text { New-onset AF } \\ \text { Age (years) } & 61[46,71] & 831 \\ \text { Male sex } & 3896(58.1) & 71[63,78] \\ \text { Severe comorbidity present } & 995(14.8) & 508(61.1) \\ \text { Surgical admission } & & 145(17.4) \\ \text { Elective surgery } & 3043(45.4) & 290(34.9) \\ \text { Admission diagnosis } & 1615(24.1) & 106(12.8) \\ \text { Medical - cardiovascular / shock } \\ \text { Surgical - gastrointestinal } \\ \text { Medical - respiratory } \\ \text { Surgical - vascular }\end{array}$




\begin{tabular}{|c|c|c|}
\hline Medical-genitourinary & $176(2.6)$ & $42(5.1)$ \\
\hline Surgical - trauma & $116(1.7)$ & $7(0.8)$ \\
\hline Other & $149(2)$ & $18(2)$ \\
\hline OASIS score & $33[24,41]$ & $42[33,49]$ \\
\hline Vasopressor use & $3086(46.0)$ & $647(77.9)$ \\
\hline Ventilated in first $24 \mathrm{~h}$ & $3689(55.0)$ & $566(68.1)$ \\
\hline Maximum HR in first 24h (bpm) & $104[90,119]$ & $114[97,133]$ \\
\hline Minimum MAP in first $24 \mathrm{~h}(\mathrm{mmHg})$ & $60[53,67]$ & $56[49,62]$ \\
\hline Maximum temperature in first $24 \mathrm{~h}\left({ }^{\circ} \mathrm{C}\right)$ & $37.1[36.7,37.7]$ & $37.2[36.8,37.8]$ \\
\hline Urine output in first $24 \mathrm{~h}(\mathrm{ml})$ & $1505[990,2300]$ & $1057[594,1630]$ \\
\hline $\begin{array}{r}\text { Plasma potassium concentration at } \\
\text { admission (mmol/L) }\end{array}$ & $4.1[3.7,4.5]$ & $4.2[3.7,4.8]$ \\
\hline $\begin{array}{r}\text { Plasma magnesium concentration at } \\
\text { admission ( } \mathrm{mmol} / \mathrm{L})\end{array}$ & $0.78[0.67,0.91]$ & $0.82[0.70,0.94]$ \\
\hline $\begin{array}{l}\text { Plasma urea concentration at admission } \\
\qquad(\mathrm{mmol} / \mathrm{L})\end{array}$ & $6.5[4.6,10.5]$ & $10.6[7.0,17.6]$ \\
\hline $\begin{array}{r}\text { Plasma creatinine concentration at } \\
\text { admission }(\mu \mathrm{mol} / \mathrm{L})\end{array}$ & $82[60,130]$ & $113[76,196]$ \\
\hline WBC at admission $\left(\times 10^{9} / \mathrm{L}\right)$ & $11.7[8.3,16.1]$ & $11.6[7.1,16.6]$ \\
\hline $\begin{array}{r}\text { Plasma lactate concentration at } \\
\text { admission (mmol/L) }\end{array}$ & $1.5[1.0,2.5]$ & $1.8[1.1,3.2]$ \\
\hline
\end{tabular}

Continuous variables are summarised as median [interquartile range], categorical variables are summarised as number (percentages).

Characteristics of new-onset atrial fibrillation

The median time from ICU admission to the first episode of NOAF was 39 hours (IQR 18-77). The median total duration of atrial fibrillation per patient was 9 hours (IQR 2-28 hours). There were no clear trends in annual incidence of atrial fibrillation over the study period (see Supplementary figure 1, Additional file 1).

Hospital outcome

Overall hospital mortality in our cohort was $17.7 \%$. This was increased in the NOAF cohort (37.1 vs $15.3 \%, p<0.001$ ). The NOAF cohort had an increased hospital rate of death per unit time (adjusted 
CSHR 1.57 (95\% Cl 1.37-1.1.81), $\mathrm{p}<0.001$ ). Patients with NOAF also had a reduced rate of hospital discharge alive per unit time (adjusted CSHR 0.68 (95\% Cl 0.63-0.73), $\mathrm{p}<0.001$ ) despite adjusting for confounding variables.

The increased duration of hospital stay in patients with NOAF combined with their increased rate of hospital death per unit time resulted in a strong prognostic association with dying in hospital despite adjusting for confounding variables (adjusted subdistribution hazard ratio (SDHR) 2.04 (1.79 - 2.32), $p<0.001)$. These findings were robust across sensitivity analyses using differing covariates, cohort and NOAF definitions (tables 2, 3 and supplementary table 3, respectively). Figure 2 is a stacked cumulative incidence curve demonstrating the association between NOAF and hospital outcomes.

Table 2 - Sensitivity analysis: Adjustment for confounding variables Model $\operatorname{SDHR}(95 \% \mathrm{Cl})$

M1 (primary analysis): Sex, OASIS score and comorbidity-adjusted $2.04(1.79-2.32)$

M2: OASIS score-adjusted $2.07(1.82-2.35)$

M3: Sex, OASIS score, comorbidity and laboratory data-adjusted $1.94(1.70-2.21)$

M4: Sex, OASIS score, comorbidity, laboratory data and vasopressor use$1.85(1.63-2.11)$ adjusted

Table 3 - Sensitivity analysis: Cohort definitions by ICU LOS

\begin{tabular}{lc}
\hline ICU LOS & SDHR $(95 \% \mathrm{CI})$ \\
\hline$>24 \mathrm{~h}$ (primary analysis) & $2.04(1.79-2.32)$ \\
$>48 \mathrm{~h}$ & $2.22(1.93-2.56)$ \\
$>72 \mathrm{~h}$ & $2.29(1.97-2.67)$
\end{tabular}

We analysed the effect of the long study period on ICU mortality. Crude hospital mortality was increased in the NOAF cohort during the early (2008-2010) and late (2013-2015) phases of the study (38.8\% vs $15.8 \%$ and $35.6 \%$ vs $11.4 \%$ respectively. 


\section{Duration of AF and hospital outcomes}

We analysed the independent impact of NOAF of different durations on hospital outcome. NOAF of any duration was significantly associated with an increased risk of hospital mortality (fig. 3a). After adjustment for confounding factors, NOAF of over 30 minutes duration was significantly associated with an increased risk of hospital mortality (fig. 3b). Odds ratios and patient numbers per group are displayed in Supplementary table 4, Additional file 1.

\section{Discussion}

Our study shows that patients who develop AF on the ICU are significantly more likely to die in hospital than those who do not. This association persisted despite adjustment for known confounders. Aetiologically, new-onset AF was independently associated with both an increased mortality rate and reduced rate of discharge per unit time. Patients who develop NOAF on the ICU are therefore exposed to an increased death rate for a longer period, resulting in a significant overall risk of dying in hospital. This study also demonstrates the association of short episodes of NOAF with hospital mortality despite adjustment for confounding variables.

To the best of our knowledge, our study is the largest to date using multivariable survival analysis to assess independent prognostic associations between NOAF and hospital outcomes. Several previous studies have suggested an independent association between NOAF during critical illness and hospital mortality using logistic regression analysis [14, 16-18]. However, logistic regression may underestimate the association between NOAF and ICU mortality due to immortal time bias [37, 39, 50]. We treated NOAF as a time-varying covariate in our primary analysis to mitigate this issue and reveal a stronger association than has been demonstrated in studies using logistic regression. Where the overall probability of an event is low (i.e. $<0.2$ ), the SDHR can be interpreted as an odds ratio for the cumulative incidence of an event [51]. Thus acquiring NOAF is associated with an approximate doubling of the odds of dying in hospital. Our results are consistent with the one smaller study investigating ICU outcomes that used survival analysis [13]. 
We analysed the effect of NOAF on hospital length of stay using competing risk methodology [52]. Previous studies report an independent association between NOAF and prolonged hospital LOS [12, 16].As developing NOAF is associated with an increase in the rate of in-hospital death per unit time, the average hospital length of stay will be shortened in the NOAF cohort. Studies that do not treat hospital discharge as a competing risk to mortality will therefore underestimate the effect of NOAF on prolonging hospital length of stay in those patients that remain alive. We demonstrated that NOAF significantly reduces the chance of hospital discharge alive at any given time. NOAF will impact on discharge decisions and an independent association with increased hospital LOS is probable and supported by our results.

There are convincing mechanistic explanations to provide biological plausibility to an independent association of NOAF with adverse hospital outcomes. Organised atrial activity contributes to ventricular filling, cardiac output and the closure of the atrioventricular valves [53, 54]. NOAF precludes these mechanisms and is temporally associated with a reduction in cardiac index [55]. These effects may explain observations that NOAF precedes haemodynamic instability and organ failure in critically ill patients [6]. NOAF is also associated with early thromboembolic complications during critical illness that may contribute to morbidity and mortality [56]. Any detrimental effect on haemodynamics may have considerable impact in any critically ill patient with minimal physiological reserve.

We identified an independent increased mortality risk associated with episodes of NOAF of any duration over 30 minutes. Atrial contractile dysfunction has been demonstrated after AF episodes of one hour [57] and can last for several weeks after cardioversion [58]. This phenomenon may occur partly due to impaired cellular calcium handling $[59,60]$ and occurs after spontaneous, chemical and electrical cardioversion $[61,62]$. Our findings may be in part explained by these deleterious effects of AF persisting beyond restoration of sinus rhythm in those patients with brief episodes of AF. 
Our study has some limitations. The study was retrospective in nature and the development of NOAF was not independently verified. Our dataset contained comprehensive data relating to severe comorbidities including severe cardiovascular disease. We were unable to correct for more common, less severe comorbidities. However, the effect of these comorbidities on ICU outcome appears to be of less importance than physiological measurements [63]. Furthermore, the development of NOAF on the ICU is more strongly linked with acute factors rather than pre-existing diagnoses $[7,13]$. We therefore do not feel our results would have been altered significantly had more extensive comorbidity data been available. Our dataset contained patients with missing hospital outcome data. It is unlikely that this would introduce any bias into the results as the data were missing at random and represented a relatively small proportion of the data compared to recent similar publications [14]. We did not analyse the impact of different NOAF treatments on outcomes as our interest lay in the prognostic value of acquiring NOAF, regardless of management. The long study period may have introduced bias from changes in practice. However our analysis revealed that the rates of NOAF and its associated mortality has persisted over time, in the context of gradual improvements in hospital survival in NOAF and never AF cohorts.

Comprehensive medication history data obtained by pharmacists were available allowing us to identify patients on antiarrhythmic or anticoagulant medications with an AF indication prior to admission. We cannot however rule out misclassification regarding patients' AF status in those rare patients without prescriptions for these medications who were not in AF in the first hours of admission.

\section{Conclusions}

We present the largest study using multivariable survival analysis to demonstrate the association between NOAF acquired in ICU and hospital outcomes. This study demonstrates that patients who develop NOAF in an ICU are significantly more likely to die in hospital than those who do not, independent of other predictors. Our study also clarifies the association between AF burden and 
Hospital outcomes. Further work is needed to investigate modifiable risk factors and optimal management strategies.

\section{Declarations}

\section{Ethics approval and consent to participate}

The PICRAM study was granted ethical approval by the NRES Committee Oxford (ref: 11/SC/0440)

and the National Information Governance Board (ref: ECC 7-05(f)/2011).

\section{Consent for publication}

Not applicable

\section{Availability of data and materials}

The PICRAM dataset is not publicly available. Whilst de-identified, it may still contain indirect

identifiers. It is therefore not currently appropriate for public dissemination.

\section{Funding}

JB is funded by an NIHR Doctoral Fellowship.

RH is funded by an NIHR Doctoral Fellowship.

SG is funded by an NIHR Doctoral Fellowship.

PW is supported by the NIHR Biomedical Research Centre, Oxford.

The PICRAM database was created with support from the Health Innovation Challenge Fund (HICF0510-006; WT-094951), a parallel funding partnership between the Department of Health and Wellcome Trust. Access was granted by the owners of the research database, the University of Oxford Critical Care Research Group.

These funders were not involved in study design or reporting. 


\section{Competing interests}

Prof. Young's institution received funding from Health Innovation Challenge Fund (joint venture of

Wellcome Trust and U.K. Department of Health. Prof. Watkinson's institution received funding from Drayson Health and National Institute for Health Research (NIHR) Biomedical Research Centre,

Oxford. Prof. Watkinson has developed an electronic observations application for which Drayson Health (now Sensyne Health) has purchased a sole license. The company has a research agreement with the University of Oxford and pay personal fees. Prof. Watkinson owns shares in Drayson Health. No organisations played a role in the study process.

No other authors have financial relationships with any organisations that might have an interest in the submitted work in the previous 3 years, nor other relationships or activities that could appear to have influenced the submitted work. Dr Hatch, Dr Bedford, Dr Rechner and Mr Gerry have declared they do not have any potential conflicts of interest.

\section{Authors' contributions}

All authors contributed substantially to the conception and design of this study. JB had access to all the data in the study and takes responsibility for the integrity of the data and accuracy of the analysis. JB, RH, SG, IR, DY and PW drafted the manuscript and actively contributed to revisions. All authors gave final approval of this version to be submitted.

\section{Acknowledgements}

Not applicable

\section{References}

[1] Artucio H, Pereira M. Cardiac arrhythmias in critically ill patients: epidemiologic study. Crit Care Med 1990;18(12):1383-8.

[2] Knotzer H, Mayr A, Ulmer H, Lederer W, Schobersberger W, Mutz N, et al. Tachyarrhythmias in a surgical intensive care unit: a case-controlled epidemiologic study. Intensive care medicine 2000;26(7):908-14.

[3] Burris JM, Subramanian A, Sansgiry S, Palacio CH, Bakaeen FG, Awad SS. Perioperative atrial arrhythmias in noncardiothoracic patients: a review of risk factors and treatment strategies in the veteran population. American journal of surgery 2010;200(5):601-5.

[4] Brathwaite D, Weissman C. The new onset of atrial arrhythmias following major noncardiothoracic surgery is associated with increased mortality. Chest 1998;114(2):462-8. 
[5] Walkey AJ, Greiner MA, Heckbert SR, Jensen PN, Piccini JP, Sinner MF, et al. Atrial fibrillation among Medicare beneficiaries hospitalized with sepsis: incidence and risk factors. American heart journal 2013;165(6):949-55 e3.

[6] Kanji S, Williamson DR, Yaghchi BM, Albert M, McIntyre L, Canadian Critical Care Trials G. Epidemiology and management of atrial fibrillation in medical and noncardiac surgical adult intensive care unit patients. Journal of critical care 2012;27(3):326 e1-8.

[7] Kuipers S, Klein Klouwenberg PM, Cremer OL. Incidence, risk factors and outcomes of newonset atrial fibrillation in patients with sepsis: a systematic review. Crit Care 2014;18(6):688.

[8] Seguin P, Signouret T, Laviolle B, Branger B, Malledant $Y$. Incidence and risk factors of atrial fibrillation in a surgical intensive care unit. Crit Care Med 2004;32(3):722-6.

[9] Seguin P, Laviolle B, Maurice A, Leclercq C, Malledant Y. Atrial fibrillation in trauma patients requiring intensive care. Intensive care medicine 2006;32(3):398-404.

[10] Gupta S, Tiruvoipati R, Green C. Atrial Fibrillation and Mortality in Critically III Patients: A Retrospective Study. Am J Crit Care 2015;24(4):336-41.

[11] Makrygiannis SS, Rizikou D, Patsourakos NG, Lampakis M, Margariti A, Ampartzidou OS, et al. New-onset atrial fibrillation and clinical outcome in non-cardiac intensive care unit patients. Aust Crit Care 2018;31(5):274-7.

[12] Carrera P, Thongprayoon C, Cheungpasitporn W, lyer VN, Moua T. Epidemiology and outcome of new-onset atrial fibrillation in the medical intensive care unit. Journal of critical care 2016;36:102-6.

[13] Klein Klouwenberg PM, Frencken JF, Kuipers S, Ong DS, Peelen LM, van Vught LA, et al. Incidence, Predictors, and Outcomes of New-Onset Atrial Fibrillation in Critically III Patients with Sepsis. A Cohort Study. Am J Respir Crit Care Med 2017;195(2):205-11.

[14] Arrigo M, Ishihara S, Feliot E, Rudiger A, Deye N, Cariou A, et al. New-onset atrial fibrillation in critically ill patients and its association with mortality: A report from the FROG-ICU study. Int J Cardiol 2018;266:95-9.

[15] Chen AY, Sokol SS, Kress JP, Lat I. New-onset atrial fibrillation is an independent predictor of mortality in medical intensive care unit patients. The Annals of pharmacotherapy 2015;49(5):523-7.

[16] Moss TJ, Calland JF, Enfield KB, Gomez-Manjarres DC, Ruminski C, DiMarco JP, et al. NewOnset Atrial Fibrillation in the Critically III. Crit Care Med 2017;45(5):790-7.

[17] Shaver CM, Chen W, Janz DR, May AK, Darbar D, Bernard GR, et al. Atrial Fibrillation Is an Independent Predictor of Mortality in Critically III Patients. Crit Care Med 2015;43(10):210411.

[18] Walkey AJ, Wiener RS, Ghobrial JM, Curtis LH, Benjamin EJ. Incident stroke and mortality associated with new-onset atrial fibrillation in patients hospitalized with severe sepsis. JAMA 2011;306(20):2248-54.

[19] Walkey AJ, Hammill BG, Curtis LH, Benjamin EJ. Long-term outcomes following development of new-onset atrial fibrillation during sepsis. Chest 2014;146(5):1187-95.

[20] Johnson AE, Burgess J, Pimentel MA, Clifton DA, Young JD, Watkinson PJ, et al. Physiological trajectory of patients pre and post ICU discharge. Conf Proc IEEE Eng Med Biol Soc 2014;2014:3160-3.

[21] von Elm E, Altman DG, Egger M, Pocock SJ, Gotzsche PC, Vandenbroucke JP, et al. Strengthening the Reporting of Observational Studies in Epidemiology (STROBE) statement: guidelines for reporting observational studies. BMJ 2007;335(7624):806-8.

[22] Palmer E, Post B, Klapaukh R, Marra G, MacCallum NS, Brealey D, et al. The Association between Supraphysiologic Arterial Oxygen Levels and Mortality in Critically III Patients. A Multicenter Observational Cohort Study. Am J Respir Crit Care Med 2019;200(11):1373-80.

[23] Yoshida T, Uchino S, Yokota T, Fujii T, Uezono S, Takinami M. The impact of sustained newonset atrial fibrillation on mortality and stroke incidence in critically ill patients: A retrospective cohort study. Journal of critical care 2018;44:267-72. 
[24] Wetterslev M, Haase N, Hassager C, Belley-Cote EP, McIntyre WF, An Y, et al. New-onset atrial fibrillation in adult critically ill patients: a scoping review. Intensive care medicine 2019;45(7):928-38.

[25] Kirchhof P, Benussi S, Kotecha D, Ahlsson A, Atar D, Casadei B, et al. 2016 ESC Guidelines for the management of atrial fibrillation developed in collaboration with EACTS. Eur Heart J 2016;37(38):2893-962.

[26] Zhang Z. Multiple imputation with multivariate imputation by chained equation (MICE) package. Ann Transl Med 2016;4(2):30.

[27] R Core Team. R: A Language and Environment for Statistical Computing. Vienna, Austria: R Foundation for Statistical Computing; 2017.

[28] Harrison DA, Ferrando-Vivas P, Shahin J, Rowan KM. Ensuring comparisons of health-care providers are fair: development and validation of risk prediction models for critically ill patients. Southampton (UK); 2015.

[29] Johnson AE, Kramer AA, Clifford GD. A new severity of illness scale using a subset of Acute Physiology And Chronic Health Evaluation data elements shows comparable predictive accuracy. Crit Care Med 2013;41(7):1711-8.

[30] Altman DG, McShane LM, Sauerbrei W, Taube SE. Reporting recommendations for tumor marker prognostic studies (REMARK): explanation and elaboration. BMC Med 2012;10:51.

[31] Bakker J, Coffernils M, Leon M, Gris P, Vincent JL. Blood lactate levels are superior to oxygenderived variables in predicting outcome in human septic shock. Chest 1991;99(4):956-62.

[32] Pirracchio R, Petersen ML, Carone M, Rigon MR, Chevret S, van der Laan MJ. Mortality prediction in intensive care units with the Super ICU Learner Algorithm (SICULA): a population-based study. Lancet Respir Med 2015;3(1):42-52.

[33] Upala S, Jaruvongvanich V, Wijarnpreecha K, Sanguankeo A. Hypomagnesemia and mortality in patients admitted to intensive care unit: a systematic review and meta-analysis. QJM : monthly journal of the Association of Physicians 2016;109(7):453-9.

[34] Brivet FG, Kleinknecht DJ, Loirat P, Landais PJ. Acute renal failure in intensive care units-causes, outcome, and prognostic factors of hospital mortality; a prospective, multicenter study. French Study Group on Acute Renal Failure. Crit Care Med 1996;24(2):192-8.

[35] Augusto JB, Fernandes A, Freitas PT, Gil V, Morais C. Predictors of de novo atrial fibrillation in a non-cardiac intensive care unit. Rev Bras Ter Intensiva 2018;30(2):166-73.

[36] Fernando SM, Mathew R, Hibbert B, Rochwerg B, Munshi L, Walkey AJ, et al. New-onset atrial fibrillation and associated outcomes and resource use among critically ill adults-a multicenter retrospective cohort study. Crit Care 2020;24(1):15.

[37] Shintani AK, Girard TD, Eden SK, Arbogast PG, Moons KG, Ely EW. Immortal time bias in critical care research: application of time-varying Cox regression for observational cohort studies. Crit Care Med 2009;37(11):2939-45.

[38] Walkey AJ, McManus D. When Rhythm Changes Cause the Blues: New-Onset Atrial Fibrillation during Sepsis. Am J Respir Crit Care Med 2017;195(2):152-4.

[39] Ho AM, Dion PW, Ng CS, Karmakar MK. Understanding immortal time bias in observational cohort studies. Anaesthesia 2013;68(2):126-30.

[40] Austin PC, Lee DS, Fine JP. Introduction to the Analysis of Survival Data in the Presence of Competing Risks. Circulation 2016;133(6):601-9.

[41] Wolkewitz $\mathrm{M}$, Sommer H. Evaluating mortality in an intensive care unit requires extended survival models. Crit Care 2014;18(2):415.

[42] Resche-Rigon M, Azoulay E, Chevret S. Evaluating mortality in intensive care units: contribution of competing risks analyses. Crit Care 2006;10(1):R5.

[43] Gooley TA, Leisenring W, Crowley J, Storer BE. Estimation of failure probabilities in the presence of competing risks: new representations of old estimators. Stat Med 1999;18(6):695-706. 
[44] Wolkewitz M, Vonberg RP, Grundmann H, Beyersmann J, Gastmeier P, Barwolff S, et al. Risk factors for the development of nosocomial pneumonia and mortality on intensive care units: application of competing risks models. Crit Care 2008;12(2):R44.

[45] Feakins BG, McFadden EC, Farmer AJ, Stevens RJ. Standard and competing risk analysis of the effect of albuminuria on cardiovascular and cancer mortality in patients with type 2 diabetes mellitus. Diagn Progn Res 2018;2:13.

[46] Noordzij M, Leffondre K, van Stralen KJ, Zoccali C, Dekker FW, Jager KJ. When do we need competing risks methods for survival analysis in nephrology? Nephrol Dial Transplant 2013;28(11):2670-7.

[47] Lau B, Cole SR, Gange SJ. Competing risk regression models for epidemiologic data. Am J Epidemiol 2009;170(2):244-56.

[48] Koller MT, Raatz H, Steyerberg EW, Wolbers M. Competing risks and the clinical community: irrelevance or ignorance? Stat Med 2012;31(11-12):1089-97.

[49] Fine JP, Gray RJ. A proportional hazards model for the subdistribution of a competing risk. J Am Stat Assoc 1999;94(446):496-509.

[50] Lu Y, Chen T. New-onset atrial fibrillation can be falsely associated with increased length of stay in ICU due to immortal time bias. Crit Care 2020;24(1):40.

[51] Austin PC, Fine JP. Practical recommendations for reporting Fine-Gray model analyses for competing risk data. Stat Med 2017;36(27):4391-400.

[52] Brock GN, Barnes C, Ramirez JA, Myers J. How to handle mortality when investigating length of hospital stay and time to clinical stability. BMC Med Res Methodol 2011;11:144.

[53] Samet P, Bernstein W, Levine S. Significance of the Atrial Contribution to Ventricular Filling. Am J Cardiol 1965;15:195-202.

[54] Mitchell JH, Gilmore JP, Sarnoff SJ. The transport function of the atrium. Factors influencing the relation between mean left atrial pressure and left ventricular end diastolic pressure. Am J Cardiol 1962;9:237-47.

[55] Pozzoli M, Cioffi G, Traversi E, Pinna GD, Cobelli F, Tavazzi L. Predictors of primary atrial fibrillation and concomitant clinical and hemodynamic changes in patients with chronic heart failure: a prospective study in 344 patients with baseline sinus rhythm. J Am Coll Cardiol 1998;32(1):197-204.

[56] Champion S, Lefort Y, Gauzere BA, Drouet D, Bouchet BJ, Bossard G, et al. CHADS2 and CHA2DS2-VASc scores can predict thromboembolic events after supraventricular arrhythmia in the critically ill patients. Journal of critical care 2014;29(5):854-8.

[57] Louie EK, Liu D, Reynertson SI, Loeb HS, McKiernan TL, Scanlon PJ, et al. "Stunning" of the left atrium after spontaneous conversion of atrial fibrillation to sinus rhythm: demonstration by transesophageal Doppler techniques in a canine model. J Am Coll Cardiol 1998;32(7):2081-6.

[58] Manning WJ, Silverman DI, Katz SE, Riley MF, Come PC, Doherty RM, et al. Impaired left atrial mechanical function after cardioversion: relation to the duration of atrial fibrillation. J Am Coll Cardiol 1994;23(7):1535-40.

[59] Sun H, Gaspo R, Leblanc N, Nattel S. Cellular mechanisms of atrial contractile dysfunction caused by sustained atrial tachycardia. Circulation 1998;98(7):719-27.

[60] Sanders P, Morton JB, Morgan JG, Davidson NC, Spence SJ, Vohra JK, et al. Reversal of atrial mechanical stunning after cardioversion of atrial arrhythmias: implications for the mechanisms of tachycardia-mediated atrial cardiomyopathy. Circulation 2002;106(14):180613.

[61] Mattioli AV, Castelli A, Andria A, Mattioli G. Clinical and echocardiographic features influencing recovery of atrial function after cardioversion of atrial fibrillation. Am J Cardiol 1998;82(11):1368-71.

[62] Shapiro EP, Effron MB, Lima S, Ouyang P, Siu CO, Bush D. Transient atrial dysfunction after conversion of chronic atrial fibrillation to sinus rhythm. Am J Cardiol 1988;62(17):1202-7. 
[63] Harrison DA, Parry GJ, Carpenter JR, Short A, Rowan K. A new risk prediction model for critical care: the Intensive Care National Audit \& Research Centre (ICNARC) model. Crit Care Med 2007;35(4):1091-8.

\section{Figure captions}

\section{Figure 1 - Study flowchart}

Figure 2 - Stacked cumulative incidence curves for each cohort. These curves demonstrate the rates of discharge alive and hospital death for patients who never had AF and patients with NOAF. The cumulative incidence curves for each event are stacked vertically for each cohort. Each stacked graph will reach 1 when every patient has either been discharged or has died.

Figure 3 - Odds ratios for hospital mortality associated with NOAF of different durations. Panel A shows unadjusted estimates, panel B shows estimates after adjustment for confounding variables.

\section{Additional files}

File name: Additional file 1

File format: .pdf

Title of data: Supplementary material

Description of data: Supplementary material 